\title{
Musculoskeletal performance and hydration status
}

\author{
Joseph A. Rothenberg · André Panagos
}

Published online: 4 March 2008

(C) Humana Press 2008

\begin{abstract}
Maximal performance during competition is the drive many competitors use to train harder. However, there are several variables that contribute to impair a competitor's performance. These variables work by altering the homeostatic mechanisms within the body. Once homeostasis is altered the competitor's body is no longer optimized to face the stresses of the athletic competition. The environment works as an all encompassing variable that will affect sweat rate. During increased environmental heat strain, one must adjust for critical variables, such as temperature regulation, hydration status, and electrolyte levels, as they can contribute to impaired performance. Acclimatization through training and competition will reduce or slow down the effects of these stress factors. Ever evolving recommendations are produced to aid competitors in maintaining homeostasis. Despite all the generic recommendations that are made, however, every athlete needs to individualize their training and competition regimens to optimize personal performance.
\end{abstract}

Keywords Hydration status - Hyponatremia · Dehydration

\section{Introduction}

Athletes continuously look for ways to improve performance. This drive goes from the elite athlete to the weekend warrior. Whether it is to run faster, longer, or simply take their body further than they have before, there

J. A. Rothenberg $(\bowtie) \cdot$ A. Panagos

Physical Medicine \& Rehabilitation, New York Presbyterian

Hospital, New York, NY, USA

e-mail: jr872-60@mindspring.com are always some goals to be met and surpassed. Most ergogenic aids are designed to help the competitor maintain homeostasis the longest, as when normal metabolism becomes disturbed that performance declines. The muscles are the tools we use to perform these tasks, and it is the fluid in the body that carries needed nutrients, which act as catalysts to keep us moving. Therefore, hydration status and maintenance of homeostasis are critical factors before, during, and after competition.

The typical person is composed of approximately $60 \%$ water, with highly trained athletes approaching $70 \%$ water content throughout their bodies [1]. This increased total body water (TBW) content in athletes is typically commensurate to their relative lean body mass compared to the general population. Approximately $65 \%$ of TBW is intracellular, while $35 \%$ is extracellular (comprising plasma and interstitial fluid) [2].

Euhydration is defined as the normal state of water content in the absence of relative dehydration [3]. Dehydration is defined as the condition that results from the loss of excessive body water, with amounts greater than $2 \%$ being considered significant [4]. Dehydration reduces endurance exercise performance, impairs exercise tolerance, and can result in hyperthermia [5]. Dehydration of $\sim 2 \%$ results in symptoms of thirst, flushing, dark-colored urine, dry mouth, fatigue, and weakness. After 5\% dehydration sweating decreases and core temperature rises. A $10 \%$ loss of body water is considered an emergency with symptoms including muscle spasms, confusion, and potential loss of consciousness [6].

In conjunction, thermoregulation is also important as it can be drastically affected by hydration status. Thermoregulation is affected by metabolism, radiation, conduction, convection, and evaporation [7]. Radiation is the loss/gain of heat in the form of infrared energy. Conduction is the 
Table 1 Progressive symptoms of dehydration during exercise [6]

\begin{tabular}{ll}
\hline Percent dehydration $(\%)$ & Typical symptoms \\
\hline 2 & Thirst, skin flushing, fatigue, weakness, dark-colored urine \\
5 & Decreased sweating, increased core temperature, increased heart and respiratory rate \\
10 & Muscle spasms, confusion, seizures, potential loss of consciousness \\
\hline
\end{tabular}

transfer of energy down a thermal gradient. Convection is the transfer of energy through moving gases or liquids. Evaporation is the heat loss through insensible water loss and sweating. At rest, evaporation accounts for $\sim 25 \%$ of heat loss, with $2.43 \mathrm{~kJ}$ of heat lost for every milliliter of water that evaporates [8]. This percentage significantly increases with exercise (Table 1).

Despite strong research that dehydration will cause decrements in endurance performance, fluid needs during competition are widely variable. These variables include, but are not limited to the type of sport, competitor experience, and environmental factors [6]. The adequate intake of total water for young men and women is considered to be 2.7-3.7 1 daily [9]. However, this reference does not account for water loss through sweat during activity, which has been documented as $1.0-2.5 \mathrm{l} / \mathrm{h}$ or greater, and can exceed $10 \mathrm{l}$ daily $[10,11]$.

In comparison to endurance-related activities, limited research on high-intensity anaerobic activities reveals that performance is not adversely affected by moderate dehydration or moderate hyperthermia [12, 13]. The theorized reasoning is that the heat strain associated with these short duration activities is not sufficient to cause performance decrements. Therefore, it is the heat strain with continued activity that at least partially contributes to performance impairments.

Numerous hydration supplements are marketed with claims to improve athletic performance. This marketing even solicits water, both plain and flavored. Most athletes have heard of and probably sampled various carbohydrate and electrolyte beverages. Data for hydration aids-containing glycerol to improve pre-competition hyperhydration are limited [8]. There are some "sports drinks" that are being marketed because of their protein content and other ergogenic aids, such as various herbs and stimulants. This review will focus on macronutrients and electrolytes associated with hydration supplementation. There is limited research on some of the fringe supplements such as stimulants and herbs. Overall, there is little consensus on the optimal fluid replacement beverage.

Training or competition of high-intensity sports such as soccer, basketball, and tennis have relatively mild to moderate dehydration levels with voluntary fluid intake [6]. Participants in these events may benefit from sports drinks by optimizing hydration through improved palatability [14, 15]. However, it is important that these drinks do not contain excessive concentrations of carbohydrate, as this could decrease performance secondary to gastrointestinal distress [16]. Although modest, Shi et al. in 2004, revealed that carbohydrate drinks with concentrations as low as $8 \%$ resulted in gastrointestinal distress and impaired performance during high-intensity intermittent exercise [17].

Other than for improved palatability, there are insufficient data to support the consumption of carbohydrate drinks by athletes competing and training less than $1 \mathrm{~h}$, with the underlying assumption that they have optimized hydration during these events and have started these events both euhydrated and with optimized meal schedules. Gastric emptying studies have shown that ingested carbohydrate solutions reach peak intestinal absorption in 20-30 min, with longer periods of absorption for greater concentrations of carbohydrates or proteins [18]. The addition of electrolytes to a carbohydrate-based solution has not been shown to enhance either gastric or intestinal absorption [19]. Gastric emptying is also related to activity intensity, with greater intensity leading to delayed absorption [20]. Given the delayed gastric emptying associated with protein drinks, and the propensity for gastrointestinal distress that can occur at higher concentration solutions and increasing exercise intensities, there are relatively few times when protein-based hydration solutions should be recommended during activity.

Basically, from a physiologic perspective, water is the optimal hydration strategy for activities lasting less than $1 \mathrm{~h}$. Post-exercise rehydration is recommended for any fluid deficits that occur during activity. However, in real world situations, the likelihood that athletes will be able to sufficiently rehydrate on plain water alone between events is remote given the highly competitive nature of sports such as soccer and athletes' condensed training schedules [21]. Although data are limited, personal experience reveals that many athletes start off an event or training day at less than optimal hydration status. Therefore, individualization for athletes based on exercise intensity, sport, sweat rate, and environmental factors is recommended.

\section{Environmental factors}

"Water, water, everywhere, nor any drop to drink." This quote by Samuel Taylor Coleridge in The Rime of the Ancient Mariner though written over 200 years ago can be 
related to the importance of the environment during sports competitions [22]. The risk of heat illness during high intensity or endurance activities is significantly increased in hot and/or humid environments [5]. Acclimatization to hot and/or humid conditions is generally noted to take 10 14 days [5]. However, benefits have been noted in 56 days with more time needed to acclimatize in humid environments [23, 24]. This adjustment to extreme temperatures enables the highly trained competitor to tolerate adverse conditions longer than someone who has not acclimatized.

The deleterious effects of a hot environment were notable during the 2007 Chicago Marathon. Although most of the news coverage focused on the increased number of participants having heat-related illnesses, contributed to by a lack of acclimatization, even the elite runners were affected, finishing 4 min slower than in their competition just 1 year prior [25]. According to Ely et al. [26], the results of the Chicago Marathon should have been expected, with their research revealing progressive slowing of marathon performance as temperature increases. This contrasts to data collected from the 1996 Atlanta Olympic Marathon in which the hot and humid temperatures were not found to significantly affect finishing times [27]. The significance of these conflicting results is likely secondary to acclimatization. Elite competitors still suffer from the same side effects as their non-elite counterparts. These effects, however, are attenuated due to their improved fitness and their ability to finish faster, thereby reducing the overall environmental strain.

Overall, the environment plays an important role during activities. Hot environments result in significant heat stress, which is compounded by dehydration. The amount of time needed to reach exercise-related exhaustion is decreased with increasing temperature, humidity, and dehydration [5]. In contrast, the effects of dehydration on endurance exercise performance are diminished in cold compared to temperate environments [28]. The etiology of this is likely related to the increased temperature gradient associated with exercise in cool environments. As previously mentioned, radiation, conduction, and convection are significant thermoregulatory mechanisms at rest, while evaporation becomes a greater contributor with activity and increasing temperature [10]. However, this evaporative thermoregulatory mechanism is significantly impaired when the relative environmental humidity is high as the partial pressure of water in the air is closer to or at its saturation point. Activities in cooler environments rely less on evaporative thermoregulation or sweating, thereby attenuating the effects of impaired performance associated with dehydration. These continued findings point to heat strain as a significant contributing factor in exercise impairment.
Theories abound as to how the body acclimates to hot environments to attenuate the performance deficits often seen during activity with adverse environmental pressures. Sweat increases in hot environments to help maintain physiologic normal core temperatures, but if the amount and concentrations do not change then the person will quickly become dehydrated and have electrolyte imbalances. In addition to the thermoregulatory effects, hydration status also affects hemodynamic stability. Dehydration can result in tachycardia through impaired plasma volume and decreased maximal cardiac output, which can lead to impaired work capacity as the exercising muscles require significant blood flow during activity [10, 29]. Increasing dehydration then results in decreased sweat rates subsequently leading to increased core temperatures. As dehydration continues the athlete's body will eventually be unable to compensate for continued environmental stressors. Acclimatization allows for adjustments of how the body reacts to these stressors. One theory of acclimatization to heat stress and sweating is that the body becomes more efficient with its sweat production by having sweat glands excrete in a patchwork skin pattern to maintain thermoregulation, while minimizing fluid loss [30, 31]. Other stressors not related to heat strain are of importance as well.

Although the effect of altitude on hydration and performance is complex, it is important to note the effects of acclimatization. Significant numbers of endurance athletes train in this type of environment to improve their performance, both at sea level and in these environments themselves [31]. Altitude acclimatization has significant physiologic effects on the human body. Living or training at elevated altitude creates a relative hypoxia, which results in the stimulation of erythropoietin. This leads to increasing red blood cells, and hemoglobin. Subsequently, the body will have increased oxygen carrying capacity [32]. However, altitude taken to the extreme as in space travel can potentially have negative performance effects. Lack of gravity has been shown to cause a relative diuresis with subsequent decreases in circulating plasma volumes [33]. The future expansion of space travel will result in more people being affected by these effects of microgravity.

\section{Electrolytes}

Acclimatization during training and competition affects more than how the body maintains hydration. The body will also conserve sodium by significantly decreasing sweat sodium concentration [34]. Hyponatremia, defined as serum sodium less than $135 \mathrm{mmol}$, is one of the most dangerous ailments of athletes because if the condition is not recognized or treated incorrectly, dire consequences 
can ensue [35]. Athletes at greatest risk are those with the saltiest sweat $(>60 \mathrm{mmol} \mathrm{Na} / \mathrm{L})$ as they will lose the greatest amount of sodium per liter of sweat [36]. Although an athlete who is acclimatized to warm weather will reduce their sweat sodium losses, a typical person $(40 \mathrm{mmol} \mathrm{Na} / \mathrm{L})$ will lose almost $1 \mathrm{gm}$ of sodium per hour given a modest sweat loss of $1 \mathrm{l} / \mathrm{h}$ [37]. In the absence of other interventions there is a significant time and sweat rate factor in determining hyponatremia risk.

A second common cause of exercise-related hyponatremia occurs with the dilution effect of fluid consumption. In the 1980s and 1990s it was common to hear race organizers and even the US military recommend that athletes and recruits consume fluids in excess of rated thirst to prevent dehydration [38]. Even though these organizers were well meaning, these recommendations and guidelines failed to account for physiologic limitations. The kidneys have a water filtration limit of approximately $1 \mathrm{l} / \mathrm{h}$, however these athletes and recruits were consuming greater than $1.5 \mathrm{l} / \mathrm{h}$ [39]. This is the theorized reasoning for the increased incidence of hyponatremia during this time period. The subsequent release of the ACSM position stand on exercise and fluid replacement in 1996 (subsequently revised in 2007) partially addressed the risks and helped to reduce the incidence of hyponatremia associated with excess fluid consumption [40].

Despite these changes, Noakes and Speedy [38] reported that there is considerable risk of hyponatremia in groups exercising greater than $4 \mathrm{~h}$. The theory is that in a sport such as a typical marathon, these slower runners will consume more fluid than they sweat out and suffer from dilution hyponatremia. These concerns were directly addressed in the most recent International Marathon Medical Directors Association Position Statement 2006, which gives recommendations of fluid intake that is directly proportional to running speed [41, 42]. Although slower runners should not restrict fluid intake during competition, it is important that they do not drink excessively.

In addition to monitoring fluid intake to prevent hyponatremia, data suggest that salt supplementation may be beneficial [6]. However, there is also conflicting research that suggests that the changes associated with this type of supplementation are insignificant [43]. Therefore, the direct etiology for this practice and its efficacy are not completely clear. Anecdotally, the practice continues to be recommended as it increases thirst and subsequently improves hydration status throughout the competition. Moreover, popular sports drinks do not contain sufficient concentrations of sodium to replace sweat losses [44].

As greater numbers compete in endurance events, there have also been reports of increasing numbers of medication-associated causes of hyponatremia. Non-steroidal anti- inflammatory medications (NSAID) and even the cyclooxygenase-2 inhibitors have been shown to significantly inhibit free water clearance by the kidneys in athletes during endurance competition [45]. This effect has been associated with serum dilution, weight gain, and hyponatremia [46]. Though the incidence of NSAID use has been found to be greater than the incidence of hyponatremia, there are positive correlations [47]. Despite previous findings and the theoretical physiologic reasoning for the association between NSAID and hyponatremia, Dumke et al. [48] refutes the significance of ibuprofen and hyponatremia in elite athletes when dose of medication is controlled. Overall, prudence would indicate instructing endurance athletes at risk for hyponatremia to avoid NSAIDs around competitions until further research is completed.

In spite of limited data, women are considered to be at a significantly greater risk to develop hyponatremia than men. The anecdotal reasoning has many sources, ranging from slower running speeds, to women being more acutely aware of maintaining adequate hydration, resulting in inadvertent over consumption of fluids [49]. In reality, there is a real theoretical physiologic reason for women to be at a greater risk. The increased estrogen level in women of childbearing age has been cited. Physiologically estrogen has been found to inhibit the sodium potassium ATPase enzyme, thus hindering the removal of potassium from brain cells, resulting in increased risk of cerebral edema associated with episodes hyponatremia [50]. Thus, though the etiologic reason for increased hyponatremia rates in women compared to men is unclear, there is an increased physiologic risk for poorer outcomes once these women have symptomatic hyponatremia.

Overall, the best treatment of hyponatremia is to prevent it from occurring through education of the athletes, coaches, and medical staff. Once a competitor is experiencing symptoms of hyponatremia such as cramps or mild changes in sensorium, giving salty foods is one of the safest treatments. Hypertonic 3\% saline is only warranted in severe symptoms of hyponatremia [51]. The use of isotonic fluid replacement is contraindicated as this may further complicate fluid overload and hyponatremia. Typically, patients will have spontaneous diuresis with resolution of symptoms and should be monitored until this time if fluid overload is suspected [52]. Later in the race, less-conditioned slower runners are at increased risk of hyperhydration and hyponatremia.

\section{Competition}

The effects of altered hydration can influence athletes cognitively in addition to physically. This can even occur 
before the level of dehydration reaches a point where the performer's physical performance would normally be affected. Mild dehydration has been attributed to increased headaches and subjective decreased performance without change in objective measurements [53]. These deleterious effects are further exacerbated with increasing core temperatures $>40 \mathrm{C}$. These subjective changes are more likely to affect team sports as this requires group cooperation. Although these changes have not been shown to increase the severity of injuries, Patel et al. [54] did show that the subjective measurements of the athletes indicated greater perceived symptoms. Therefore, the importance of preventing significant dehydration in team sports is evident before the individual members of the team would begin to show performance deficits.

This contrasts with endurance events such as marathon runners who typically compete as individuals with a set objective of competing against the clock as well as the other runners. Many of the elite runners will attempt to acclimate to the race environment by either traveling to the race destination early or training in a similar environment to acclimatize to the conditions they are likely to see [27]. These elite athletes will typically finish their competition in approximately $2 \mathrm{~h}$ for a standard marathon. Maintaining adequate hydration is the prime focus for these elite runners, to minimize heat strain-related performance deficits. The more casual runners such as those in the open marathons (New York, Chicago, etc.) are at greater risks for hyponatremia as they can potentially consume significantly more fluids than they need for such an event [55]. These risks appear to increase for athletes who require more than four hours to complete their event [38].

Ultra endurance events such as $100 \mathrm{~km}$ runners, triathletes, and long distance cyclists all experience unique risks associated with their sports. Competitors of ultra endurance runs can be subjected to significant heat strain depending on environmental factors, dehydration, and even hyponatremia if they are not able to consume enough sodium to offset sweat losses. Hyperhydration is rarely encountered during these types of events [56]. The above risks begin to increase when the athletes enter their third hour of competition. Alternatively, long distance cyclists have the benefit of always having fluids available with caution needed to prevent hyperhydration with associated side effects. Ebert et al. [57], hypothesized that dehydration would benefit cycling hill climbing by improving the power-to-weight ratio of the athletes. However, the deleterious effects of dehydration were greater than any benefit that could have resulted from the reduced weight during the competition. Triathletes experience a conglomerate of exercise-associated risks secondary to the long competition duration. As the athletes have no availability of drinkable water during that initial swim, dehydration is prevalent.
Research has shown that these same athletes have weights above baseline on completion of the bike leg of the race [6]. This actual increase in weight is related to the ad libitum fluid availability while on the bike leg. Anecdotal reports from triathletes indicate that many of these competitors also consume significant quantities of food that would cause gastrointestinal distress in most athletes, but is tolerated in this group as they train while eating these foods as well.

\section{Conclusion}

The keys to success during any competition are to individualize fluid and electrolyte consumption to prevent both dehydration and hyperhydration. A simplified adjustment is to calculate sweat loss during training runs and distribute fluid replacement during training and competition. Elite athletes can have sodium sweat concentrations and losses calculated and replaced with special individualized formulated electrolyte replacement drinks. Typical athletes can estimate that they lose a little less than $1 \mathrm{~g}$ of sodium for every liter of sweat. If they continue to experience cramping during training, they should attempt to increase electrolyte concentration to relieve symptoms and then adjust as necessary.

However, the most important key to a successful competition is to avoid any new experimental training changes on the competition day.

\section{References}

1. Benardot D. Ch 3: Fluids and electrolytes. In: Advanced sports nutrition. Champaign, IL: Human Kinetics; 2006. p. 75-100.

2. Guyton AC, Hall JE. Textbook of medical physiology. 10th ed. W.B. Saunders Company; 2000.

3. Medilexicon. http://www.medilexicon.com. Accessed 29 Sept 2007.

4. Bartok C, Schoeller DA, et al. Hydration testing in collegiate wrestlers undergoing hypertonic dehydration. Med Sci Sports Exerc 2004;36:510-7.

5. Armstrong LE, Casa DJ, et al. American college of sports medicine position stand: exertional heat illness during training and competition. Med Sci Sports Exerc 2007;39:556-72.

6. Sawka MN, Burke LM, et al. American college of sports medicine position stand: exercise and fluid replacement. Med Sci Sports Exerc 2007;39:377-90.

7. Cheuvront SN, Haymes EM. Thermoregulation and marathon running. Biological and environmental influences. Sports Med 2001;31:743-62.

8. Wendt D, Van Loon LJC, van Marken Lichtenbelt WD. Thermoregulation during exercise in the heat. Strategies for maintaining health and performance. Sports Med 2007;37:669_ 82.

9. Institute of Medicine. Water. In: Dietary reference intakes for water, sodium, chloride, potassium and sulfate. Washington, DC: National Academy Press; 2005. p. 73-185. 
10. Sawka MN, Montain SJ. Fluid and electrolyte supplementation for exercise heat stress. Am J Clin Nutr 2000;72:564S-72S.

11. Kenney WL. Dietary water and sodium requirements for active adults. GSSI Sports Sci Exchange 2004;17:1-6.

12. Judelson DA, Maresh CM, et al. Effect of hydration state on strength, power, and resistance exercise performance. Med Sci Sports Exerc 2007;39:1817-24.

13. Cheuvront SN, Carter R, et al. No effect of moderate hypohydration or hyperthermia on anaerobic exercise performance. Med Sci Sports Exerc 2006;38:1093-7.

14. Passe DH, Horn M, et al. Palatability and voluntary intake of sports beverages, diluted orange juice, and water during exercise. Int J Sport Nutr Exerc Metabolism 2004;14:272-84.

15. Casa DJ, Armstrong LE, et al. National athletic trainers' association position statement: fluid replacement for athletes. J Athl Training 2000;35:212-24.

16. Bonci L. Energy drinks: help, harm, or hype? GSSI Sports Sci Exchange 2002;15:1-4.

17. Shi X, Horn MK, et al. Gastrointestinal discomfort during intermittent high-intensity exercise: effect of carbohydrate electrolyte beverage. Int $\mathbf{J}$ Sport Nutr Exerc Metabolism 2004; 14:673-84.

18. Maughan RJ, Leiper JB, et al. Gastric emptying and fluid availability after ingestion of glucose and soy protein hydrolysyate solutions in man. Exp Physiol 2004;89:101-8.

19. Gisolfi CV, Summers RD, et al. Effect of sodium concentration in a carbohydrate-electrolyte solution on intestinal absorption. Med Sci Sport Exer 1995;27:1414-20.

20. Leiper JB, Prentice AS, et al. Gastric emptying of a carbohydrate electrolyte drink during a soccer match. Med Sci Sports Exerc 2001;33:1932-8.

21. Kirkendall DT. Creatine, carbs, and fluids: how important in soccer nutrition? GSSI Sports Sci Exchange 2004;17:1-6.

22. Keach W. The complete poems. In: Coleridge ST, editor. Penguin; 1997.

23. Shapiro Y, Kent B, et al. Physiological responses of men and women to humid and dry heat. J Appl Physiol:Respirat Environ Exercise Physiol 1980;49:1-8.

24. Avellini BA, Kamon E, et al. Physiological responses of physically fit men and women to acclimation to humid heat. J Appl Physiol:Respirat Environ Exercise Physiol. 1980;49:254-61.

25. Chicago Marathon. http://www.chicagomarathon.com/. Accessed 28 Oct 2007

26. Ely MR, Cheuvront SN, et al. Impact of weather on Marathonrunning performance. Med Sci Sports Exerc 2007;39:487-93.

27. Maughan RJ, Watson $\mathrm{P}$, et al. Heat and cold. What does the environment do to the marathon runner? Sports Med 2007;37:396-9.

28. Cheuvront SN, Carter R, et al. Hypohydration impairs endurance exercise performance in temperate but not cold air. J Appl Physiol 2005;99:1972-6.

29. Shirreffs SM, Aragon-Vargas LF, et al. The sweating response of elite professional soccer players to training in the heat. Int $\mathbf{J}$ Sports Med 2005;26:90-5.

30. Crawshaw LI. Temperature regulation in vertebrates. Ann Rev Physiol 1980;42:473-91.

31. Kamler K. Surviving the extremes. Penguin; 2004.

32. Schmitt L, Millet G, et al. Influence of "living high-training low on aerobic performance and economy of work in elite athletes. Eur J Appl Physiol 2006;97:627-36.

33. Diedrich A, Paranjape SY, et al. Plasma and blood volume in space. Med Sci 2007;334:80-5.

34. Allan JR, Wilson CG. Influence of acclimatization on sweat sodium concentration. J Appl Physiol 1971;30:708-12.
35. Dancaster CP, Whereat SJ. Fluid and electrolyte balance during the Comrades Marathon. S Afr Med J 1971;45:147-50.

36. Montain SJ, Sawka MN, Wenger CB. Hyponatremia associated with exercise:risk factors and pathogenesis. Exercise Sports Sci Rev 2001;3:113-7.

37. Murray B, Stofan J, Eichner ER. Hyponatremia in athletes. GSSI Sports Sci Exchange 2003;16:1-6.

38. Noakes TD, Speedy DB. Case proven: exercise associated hyponatraemia is due to overdrinking. So why did it take 20 years before the original evidence was accepted? $\mathrm{Br} \mathrm{J}$ Sports Med 2006;40:567-72.

39. Speedy DB, Noakes TD, et al. Response to a fluid load in athletes with a history of exercise induced hyponatremia. Med Sci Sports Exerc 2001;33:1434-42.

40. Convertino VA, Armstrong LE, et al. American college of sports medicine position stand: exercise and fluid replacement. Med Sci Sports Exerc 1996;28: i-vii.

41. Hew-Butler T, Verbalis JG, Noakes TD. Updated fluid recommendation: position statement from the international marathon medical directors association (IMMDA). Clin J Sport Med 2006;16:283-92.

42. IMMDA's revised fluid recommendations for runners and walkers.

http://www.aims-association.org/ guidelines_fluid_replacement.htm/. Accessed 31 Oct 2007.

43. Speedy DB, Thompson JMD, et al. Oral salt supplementation during ultradistance exercise. Clin J Sport Med 2002;12:279-84.

44. Speedy DB. The drinking athlete. N Z J Sport Med 1996;24:33-4.

45. Baker J, Cotter JD, et al. Effects of indomethacin and celecoxib on renal function in athletes. Med Sci Sports Exerc 2005;37:7127.

46. Page AJ, Reid SA, et al. Exercise-Associated hyponatremia, renal function, and nonsteroidal antiinflammatory drug use in an ultraendurance mountain run. Clin J Sport Med 2007;17:43-8.

47. Wharam PC, Speedy TD, et al. NSAID use increases the risk of developing hyponatremia during an ironman triathlon. Med Sci Sports Exerc 2006;38:618-22.

48. Dumke CL, Nieman DC, et al. Ibuprofen does not affect serum electrolyte concentrations after an ultradistance run. Br J Sports Med 2007;41:492-6.

49. Hew TD. Women hydrate more than men during a marathon race. Hyponatremia in the Houston marathon: A report on 60 cases. Clin J Sport Med 2005;15:148-53.

50. Ayus JC, Wheeler JM, et al. Post-operative hyponatremic encephalopathy in Menstruant women. Ann Intern Med 1992;117:891-7.

51. Hsieh M. Recommendations for treatment of hyponatremia at endurance events. Sports Med 2004;34:231-8.

52. Davis DP, Videen JS, et al. Exercise-associated hyponatremia in marathon runners: A two-year experience. J Emergency Med 2001;21:47-57.

53. Maughan RJ. Impact of mild dehydration on wellness and on exercise performance. Eur J Clin Nutr 2003;57:S19-23.

54. Patel AV, Mihalik JP, et al. Neuropsychological performance, postural stability, and symptoms after dehydration. J Athl Training 2007;42:66-75.

55. Montain SJ, Cheuvront SN, Sawka MN. Exercise associated hyponatremia:quantitative analysis to understanding the etiology. Br J Sports Med 2006;40:98-106.

56. Rehrer NJ. Fluid and electrolyte balance in ultra-endurance sport. Sports Med 2001;31:701-15.

57. Ebert TR, Martin DT, et al. Influence of hydration status on thermoregulation and cycling hill climbing. Med Sci Sports Exerc 2007;39:323-9. 